\title{
PSEUDO-INTEGRAL OPERATORS
}

\author{
BY
}

A. R. SOUROUR ${ }^{1}$

\begin{abstract}
Let $(X, \mathbb{Q}, m)$ be a standard finite measure space. $A$ bounded operator $T$ on $L^{2}(X)$ is called a pseudo-integral operator if $(T f(x)=$ $\int f(y) \mu(x, d y)$, where, for every $x, \mu(x, \cdot)$ is a bounded Borel measure on $X$. Main results: 1. A bounded operator $T$ on $L^{2}$ is a pseudo-integral operator with a positive kernel if and only if $T$ maps positive functions to positive functions. 2 . On nonatomic measure spaces every operator unitarily equivalent to $T$ is a pseudo-integral operator if and only if $T$ is the sum of a scalar and a Hilbert-Schmidt operator. 3. The class of pseudo-integral operators with absolutely bounded kernels form a selfadjoint (nonclosed) algebra, and the class of integral operators with absolutely bounded kernels is a two-sided ideal. 4. An operator $T$ satisfies $\left(T f(x)=\int f(y) \mu(x, d y)\right.$ for if $\in L^{\infty}$ if and only if there exists a positive measurable (almost-everywhere finite) function $\Omega$ such that $|(T f)(x)|<\|f\|_{\infty} \Omega(x)$ for $f \in L^{\infty}$.
\end{abstract}

1. Introduction. The present work is inspired by a paper of W. B. Arveson ([2], especially $\$ 1.5$ on pseudo-integral operators) and by the lectures on integral operators given by P. R. Halmos at the Conference on Concrete Representations of Operators, Long Beach, June 1977.

Let $(X, Q, m)$ be a positive finite measure space. An operator $T$ on $L^{2}(X, m)$ is called an integral operator if there is a measurable function $k$ on $X \times X$ (called the kernel of $T$ ) such that

$$
(T f)(x)=\int k(x, y) f(y) m(d y),
$$

for almost every $x \in X$ (it is more convenient to use the notation $m(d y)$ instead of $d m(y))$. In [2], Arveson introduced a broader class of operators associated with measures, rather than functions, defined on $X \times X$. He considered certain measures $\mu$ on $X \times X$ which induce bounded operators $T_{\mu}$ on $L^{2}(X, m)$ by the equation

$$
\left\langle T_{\mu} f, g\right\rangle=\int_{X \times Y} \int_{\times Y} f(y) \bar{g}(x) \mu(d x, d y),
$$

for $f, g \in L^{2}(X, m)$, where $\langle\cdot, \cdot\rangle$ denotes the usual inner product in $L^{2}$. He also showed that such operators can be given explicity, in the case of a

Received by the editors July 24, 1978.

AMS (MOS) subject classifications (1970). Primary 47G05; Secondary 47B10.

'Supported by a National Research Council (Canada) grant. 
standard measure space, by the equation

$$
\left(T_{\mu} f\right)(x)=\int_{X} f(y) \mu^{x}(d y)
$$

for almost every $x \in X$, where $\left\{\mu^{x}\right\}$ is a certain family of measures on $X$ related to $\mu$ via the theorem on "disintegration of measures".

Our definition of pseudo-integral operators is somewhat more general than Arveson's. We take the equation (2) as our definition, where $\left\{\mu^{x}\right\}$ is any family of measures which induces a bounded operator via the equation (2), and we use the notation $\mu(x, \cdot)$ instead of $\mu^{x}(\cdot)$. The family of measures $\{\mu(x, \cdot)\}$ or the map $x \rightarrow \mu(x, \cdot)$ will be called the kernel of the operator. The operators given by the equation (1) for some measure $\mu$ on $X \times X$ will be shown to coincide with a proper subclass of the class of pseudo-integral operators, namely the subclass of operators with absolutely bounded kernels.

The class of pseudo-integral operators is quite large, and substantially larger than the class of integral operators. It includes the identity (take $\mu(x, \cdot)$ to be the point mass at $x$, or take $\mu$ on $X \times X$ to be the measure which lives on the diagonal $\{(x, x): x \in X\}$ and whose "projection" on $X$ is $m)$. It contains all multiplication operators ( $\mu$ lives on the diagonal) and all composition operators ( $\mu$ lives on the graph of a function), in particular it contains all shifts on $L^{2}(0,1), L^{2}(0, \infty)$ or $L^{2}(-\infty, \infty)$.

The purpose of this paper is to study certain aspects of pseudo-integral operators (on standard measure spaces) related to the following questions: Which operators are pseudo-integral operators? Which operators have positive kernels? (Answer: a "lot", all operators which map positive functions to positive functions, see \$3). Which operators $T$ are "always pseudo-integral"? (This means that every operator in the unitary orbit of $T$ is pseudo-integral.) Answer: very few, on a non-purely-atomic measure space, only scalars + Hilbert-Schmidt are always pseudo-integral (\$5). When is the product of two pseudo-integral operators, or the adjoint of a pseudo-integral operator, pseudo-integral? (\$4). An obvious necessary condition for an operator $T$ to be pseudo-integral is the existence of a measurable function $\Omega$ which dominates the image under $T$ of the unit ball of $L^{\infty}$ (if $\mu(x, d y)$ is the kernel, take $\Omega(x)=|\mu|(x, X))$. Is this condition sufficient? (\$6).

2. Preliminaries. In what follows $(X, Q)$ will be a standard Borel space, that is, a set $X$ and a sigma-algebra $Q$ of subsets of $X$ (called the Borel sets) such that $(X, \mathcal{Q})$ is Borel-isomorphic to a Borel subset of some complete separable metric space in its relative Borel structure (see [17], or Chapter 3 of [3]). Let $m$ be a finite positive Borel measure on $X$. The measure space $(X, Q, m)$ is then called a standard measure space. Let $(Y, \mathscr{B}, n)$ be another finite positive standard measure space. We will consider bounded linear operators from 
$L^{2}(Y, n)$ into $L^{2}(X, m)$. (Although we are mainly interested in the case $Y=X$, it is notationally more convenient to use different symbols for the domain and the target space.)

Unless otherwise indicated, the term "almost everywhere" or "a.e." will be with respect to the measure $m$ (for objects defined on $X$ ) or to $n$ (for objects defined on $Y$ ). The total variation of a measure $\mu$ on a set $E$ will be denoted by $|\mu|(E)$.

Definimion 2.1. A bounded linear operator $T$ from $L^{2}(Y, n)$ into $L^{2}(X, m)$ is called a pseudo-integral operator if $T$ is given by the equation

$$
(T f)(x)=\int f(y) \mu(x, d y), \quad m \text {-a.e., }
$$

for every $f$ in $L^{2}(Y, n)$, where, for almost every $x, \mu(x, \cdot)$ is a complex-valued Borel measure on $Y$, and, for every Borel subset $B$ of $Y$, the map $x \rightarrow \mu(x, B)$ is assumed to be a Borel function.

The map $x \rightarrow \mu(x, \cdot)$ of $X$ into the set of measures on $Y$ will be called the kernel of $T$.

REMarKs 2.2. (i) It is implicit in Definition 2.1, that $\int|f(y)||\mu|(x, d y)<\infty$ a.e., for every $f \in L^{2}(Y)$. Taking $f=1$, we see that $|\mu|(x, Y)<\infty$ a.e.

(ii) If $B \in \mathscr{B}$ and $n(B)=0$, then $|\mu|(x, B)=0$ for almost every $x$. This is just a restatement of the condition that $T f=0$ a.e. whenever $f=0$ a.e.

(iii) The kernel is uniquely determined by the operator in the sense that if $\nu(x, d y)$ satisfies $(*)$ then $\mu(x, \cdot)=\nu(x, \cdot)$ for $m$-almost every $x$. To prove this let $\left\{B_{n}\right\}$ be a countable generating and separating family of $\mathscr{B}$ (see [17]). If $\chi_{n}$ is the characteristic function of $B_{n}$, then

$$
\mu\left(x, B_{n}\right)=\left(T \chi_{n}\right)(x)=\nu\left(x, B_{n}\right)
$$

for almost every $x$. Let $X_{0}$ be a Borel subset of $X$ of $m$-measure 0 such that $\mu\left(x, B_{n}\right)=\nu\left(x, B_{n}\right)$ for $x \notin X_{0}$ and every $n$. It follows easily that $\mu(x, B)=$ $\nu(x, B)$ for every $B \in \mathscr{B}$ and $x \notin X_{0}$.

(iv) If the equation (*) gives an everywhere defined linear transformation $T$ from $L^{2}(Y)$ into $L^{2}(X)$, then $T$ is automatically bounded. The proof of the analogous statement for integral operators is part of the folklore. The same argument works here too, and goes as follows: Let $f_{n} \rightarrow 0$ in $L^{2}(Y)$. Choose a subsequence $f_{n_{i}}$ which converges almost everywhere and which is dominated by a positive function $u$ in $L^{2}(Y)$. (For example take $f_{n_{i}}$ such that $\sum_{i}\left\|f_{n_{i}}\right\|^{2}<$ $\infty$, and $u^{2}(y)=\Sigma_{i}\left|f_{n_{i}}(y)\right|^{2}$.) By the dominated convergence theorem, $T f_{n_{i}} \rightarrow 0$ a.e., and the closed graph theorem implies that $T$ is bounded.

(v) The conclusion of the preceding remark is also valid for operators from $L^{P}(Y)$ into $L^{r}(X), 1<p \leqslant \infty, 1 \leqslant r \leqslant \infty$.

(vi) When $Y$ is a compact metric space, the Borel measurability condition of $x \rightarrow \mu(x, B)$ in the definition is equivalent to the condition that $x \rightarrow$ $\mu(x, \cdot)$ be weak*-measurable, i.e., that the map 


$$
x \rightarrow \int h(y) \mu(x, d y)
$$

is Borel measurable for every $h \in C(Y)$. To prove this, first assume that $x \rightarrow \mu(x, B)$ is Borel measurable for every $B \in \mathscr{B}$. Let $\mathcal{C}$ be the class of all Borel functions $h$ for which (1) is Borel measurable, then $C$ contains all simple functions, and is closed under bounded pointwise sequential convergence, hence it contains $C(Y)$. Conversely if $x \rightarrow \mu(x, \cdot)$ is weak ${ }^{*}$-measurable, then $C$ contains $C(Y)$ and hence contains all bounded Borel functions $h$, in particular, if $h=\chi_{B}$, we have $x \rightarrow \mu(x, B)$ Borel measurable.

(vii) In Definition 2.1 we assumed that $\mu(x, B)$ is a Borel function of $x$ for every $B \in \mathscr{B}$. This condition is "almost automatically" satisfied. More precisely, if $\{\mu(x, \cdot)\}$ is a family of measures satisfying equation (*) of Definition 2.1 , then by modifying the measures $\mu(x, \cdot)$ on a subset of $X$ of $m$-measure zero we obtain a kernel with the required measurability condition. To prove this, assume that $(T f)(x)=\int f(y) \mu(x, d y)$ a.e., hence $\mu(x, B)=$ $\left(T \chi_{B}\right)(x)$ a.e., and for every $B \in \mathscr{B}$, the map $x \rightarrow \mu(x, B)$ is measurable with respect to the completion $(X, \tilde{\mathbb{Q}}, m)$ of $(X, \mathcal{Q}, m)$, and hence agrees with a Borel function outside a Borel set of $m$-measure zero. Let $\left\{B_{n}\right\}$ be a countable generating set for $\mathscr{B}$, therefore there is a Borel subset $X_{0}$ of $X$ of $m$-measure zero such that $x \rightarrow \mu\left(x, B_{n}\right)$ is a Borel function on $X \backslash X_{0}$ for every $n$. Let $\bigodot$ be the class of Borel sets for which this property holds. It is easy to see that $C$ is a $\sigma$-algebra of sets, and since it contains $\left\{B_{n}\right\}$, it must equal $\mathscr{B}$. Now, redefine $\mu(x, \cdot)$ to be the zero measure for $x \in X_{0}$.

Examples 2.3. (i) Let $Y=X$ and $\phi \in L^{\infty}(X)$. The equation $M_{\phi} f=\phi f$ defines a bounded operator $M_{\phi}$. This is a pseudo-integral operator induced by the kernel: $\mu(x, d y)=\phi(x) \delta_{x}(d y)$, where $\delta_{x}$ is the point mass at $x$. In particular, the identity $I$ is a pseudo-integral operator. On a nonatomic measure space none of these operators is an integral operator. This follows from the uniqueness of the kernel (see also [10, problem 123]).

(ii) Let $\psi$ be a measurable map from $X$ into $Y$ and assume that the equation $C_{\psi} f=f \circ \psi$ defines a bounded operator $C_{\psi}$ of $L^{2}(Y)$ into $L^{2}(X)$. Then $C_{\psi}$ is a pseudo-integral operator with kernel $\mu(x, d y)=\delta_{\psi(x)}(d y)$.

(iii) The product $M_{\phi} C_{\psi}$ is a pseudo-integral operator.

These examples show that the class of pseudo-integral operators is quite large. This suggests the question whether every operator is a pseudo-integral operator. We give three counterexamples.

EXAMPLE 2.4. Let $\Gamma$ be the unit circle (with normalized Lebesgue measure), $L^{2}=L^{2}(\Gamma)$, and $H^{2}$ the usual Hardy space. We will show that the projection $P$ of $L^{2}$ onto $\left(H^{2}\right)^{\perp}$ is not a pseudo-integral operator. Suppose, to the contrary, that $(P f)(w)=\int f(z) \mu(w, d z)$ almost everywhere. Let $e_{n}(z)=z^{n}$, and remove a set $\Gamma_{0}$ of measure zero such that 


$$
\int e_{n}(z) \mu(w, d z)= \begin{cases}e_{n}(w) & \text { if } n \leqslant 0 \\ 0 & \text { if } n>0,\end{cases}
$$

for every $w \in \Gamma \backslash \Gamma_{0}$. Choose one $w \notin \Gamma_{0}$ and let $\sigma(d z)=\mu(w, d z)$. Therefore, the Fourier transform $\hat{\sigma}$ of $\sigma$ is given by

$$
\hat{\sigma}(n)= \begin{cases}w^{n} & \text { if } n \geqslant 0, \\ 0 & \text { if } n<0 .\end{cases}
$$

The theorem of F. and M. Riesz (see [12, p. 47]) implies that $\sigma$ is absolutely continuous, and by the Riemann-Lebesgue lemma (see [13, p. 13]), $\hat{\sigma}(n) \rightarrow 0$, i.e. $1=\left|w^{n}\right| \rightarrow 0$, a contradiction.

The next example was given by P. R. Halmos, as an example of an operator which is not integral, in the aforementioned lectures at Long Beach.

EXAMPLE 2.5. Let $\left\{e_{n}: n=0,1, \ldots\right\}$ be an orthonormal basis of $L^{2}(0,1)$ which consists of uniformly bounded functions, e.g. take $\left\{e_{n}(t)\right\}$ to be $\left\{e^{i m t}\right.$ : $m \in Z\}$ arranged in a sequence. Let $\left\{h_{n}\right\}$ be another orthonormal basis with the property that $\sup _{n}\left|h_{n}(t)\right|=+\infty$ for almost every $t$, e.g., take $\left\{h_{n}\right\}$ to be the Haar basis. (This is defined as follows: in the $n$th step divide $(0,1)$ into $2^{n+1}$ equal subintervals $I_{1}=\left(0,2^{-n-1}\right), I_{2}=\left(2^{-n-1}, 2 \cdot 2^{-n-1}\right), \ldots$ etc., and take the following functions: $f_{1}$ is $2^{n / 2}$ on $I_{1},-2^{n / 2}$ on $I_{2}$, and zero everywhere else; $f_{2}$ is $2^{n / 2}$ on $I_{3},-2^{n / 2}$ on $I_{4}$, and zero everywhere else, etc. This defines $2^{n}$ members of the basis.) Let $T$ be the unitary operator determined by $T e_{n}=h_{n}$. If $T$ is a pseudo-integral operator, with kernel $\mu(x, \cdot)$, then

$$
\left|h_{n}(x)\right|=\left|\left(T e_{n}\right)(x)\right| \leqslant \int\left|e_{n}(y)\right||\mu|(x, d y) \leqslant|\mu|(x, Y),
$$

for $x$ outside some null set. But this contradicts the fact that $\sup \left|h_{n}(x)\right|=\infty$ a.e.

EXAMPLE 2.6. Let $\left\{e_{n}\right\}$ and $\left\{h_{n}\right\}$ be as in the previous example, and let $T e_{n}=n^{-1 / 4} h_{n}$. Therefore $T$ extends to a compact operator. The same argument as before shows that $T$ is not a pseudo-integral operator (observe that $\left.\left\|h_{n}\right\|_{\infty}=O\left(n^{1 / 2}\right)\right)$.

In Example 2.5 a necessary condition for an operator to be a pseudo-integral operator has been established. We isolate this fact:

THEOREM 2.7. If $T$ is a pseudo-integral operator, then there exists a positive Borel function $\Omega$ on $X$ such that

$$
|(T f)(x)| \leqslant \Omega(x) \text { a.e. }
$$

whenever $\|f\|_{\infty} \leqslant 1$.

Question. Is this condition sufficient? This will be discussed in $\$ 6$.

It has been observed by P. R. Halmos that the condition of Theorem 2.7 is a necessary condition for an operator to be an integral operator. 
3. Characterization of operators with positive kernels. An obvious necessary condition for an operator $T$ to be psendo-integral with a positive kernel is that $T$ maps positive functions to positive functions. It is somewhat surprising that this condition turns out to be also sufficient. This section is devoted to proving this fact, and its simple extension to absolutely bounded kernels. The result is another indication that there are "lots" of pseudo-integral operators.

THEOREM 3.1. Let $T$ be a bounded operator from $L^{2}(Y, n)$ to $L^{2}(X, m)$. In order for $T$ to be a pseudo-integral operator with a positive kernel, it is necessary and sufficient that $T$ be a positive operator in the sense that $T$ maps positive functions to positive functions.

We will give two different proofs. First, we note that we may assume that $Y$ is a compact metric space and the Borel structure on $Y$ is generated by its topology since every standard Borel space is Borel isomorphic to a compact metric space (see [16, p. 451] or [17]). In the first proof we use techniques similar to those used by Abrahamse and Kriete in [1].

FIRST PROOF. The necessity is obvious. To prove the sufficiency, let $C_{r}(Y)$ be the space of real-valued continuous functions on $Y$. Let $\left\{e_{n}: n=0,1\right.$, $2, \ldots\}$ be a countable linearly independent subset of positive functions in $C_{r}(Y)$ such that $e_{0}=1$, and the linear span $\mathscr{D}$ of $\left\{e_{n}\right\}$ is dense in $C_{r}(Y)$. Let $\mathscr{D}_{0}$ be the linear manifold over the rationals spanned by $\left\{e_{n}\right\}$. For every $f \in \mathscr{D}$, choose a function $\pi(T f)$ in the equivalence class $T f$ in such a way that for every $x$, the map $\phi_{x}$ defined by

$$
\phi_{x}(f)=\pi(T f)(x)
$$

is a linear map of $\mathscr{D}$ into the real numbers. This can be done by first choosing $\pi\left(T e_{n}\right)$ in any manner, and then extending linearly to $\mathscr{D}$. Remove one $m$-null set $X_{0}$ of $X$, so that $\pi(T f)(x) \geqslant 0$ for every $x \notin X_{0}$ and every nonnegative $f$ in $\mathscr{D}_{0}$.

We now show that $\phi_{x}$ is bounded on $\mathscr{D}$ for $x \notin X_{0}$. First consider $f \in \mathscr{D}_{0}$ with $-1 \leqslant f(y) \leqslant 1$, then by the positivity of $\phi_{x}$ we have $-h_{0}(x) \leqslant \phi_{x}(f) \leqslant$ $h_{0}(x)$ where $h_{0}=\pi(T 1)$. Thus $\phi_{x}$ is bounded on $\mathscr{D}_{0}$ with "norm" $h_{0}(x)$. Now if $f \in \mathscr{D}$, then for some positive integer $n, f \in \mathscr{T}_{n}$, the span of $\left\{e_{1}\right.$, $\left.e_{2}, \ldots, e_{n}\right\}$. The map $\phi_{x} \mid \Re_{n}$ is linear, hence bounded, and its norm is determined by the dense set $\mathscr{D}_{0} \cap \mathfrak{T}_{n}$, so $\left|\phi_{x}(f)\right|<h_{0}(x)\|f\|_{\infty}$. This proves the boundedness of $\phi_{x}$ on $\mathscr{D}$. Therefore $\phi_{x}$ extends to a bounded linear functional on $C_{r}(Y)$, with norm $h_{0}(x)$.

Furthermore, every $\phi_{x}$ (for $x \notin X_{0}$ ) is positive, since $\left\|\phi_{x}\right\|=\phi_{x}(1)$. By the Riesz Representation Theorem, there exists a positive measure $\mu(x, \cdot)$ on $Y$ such that

$$
\pi(T f)(x)=\int f(y) \mu(x, d y) \text { for } f \in \mathscr{D}, x \in X \backslash X_{0} .
$$


If $x \in X_{0}$, let $\mu(x, \cdot)=0$, and let $\bigodot$ be the class of all $f \in L^{2}(Y, n)$ for which

$$
(T f)(x)=\int f(y) \mu(x, d y) \text { for } m \text {-almost every } x .
$$

We know that $\mathscr{D} \subset \mathcal{C}$. Next, we show that $\mathcal{C}$ contains $C(Y)$, the space of complex-valued continuous functions on $Y$. Let $f \in C_{r}(Y)$ and choose $f_{n} \in$ (1) so that $f_{n} \rightarrow f$ uniformly. By the dominated convergence theorem $\int f_{n}(y) \mu(x, d y) \rightarrow \int f(y) \mu(x, d y)$ for every $x$. Also $\left\|f_{n}-f\right\|_{2} \rightarrow 0$, hence $\| T f_{n}-$ $T f \|_{2} \rightarrow 0$, and there is a subsequence $f_{n(k)}$, so that $T f_{n(k)} \rightarrow T f$ a.e. This establishes (*) for $f \in C_{r}(Y)$ and hence for every $f \in C(Y)$.

Next, observe that $C$ is a monotone class, i.e., if $f_{n} \in \mathcal{C}, f_{n} \geqslant 0, f_{n} \uparrow f$ a.e., and $f \in L^{2}(Y, n)$, then $f \in \mathcal{C}$. To prove this use the monotone convergence theorem once to show that $\int f(y) \mu(x, d y)=\lim \int f_{n}(y) \mu(x, d y)$, then a second time to show that $\left\|f-f_{n}\right\|_{2} \rightarrow 0$, hence $\left\|T f-T f_{n}\right\| \rightarrow 0$ and therefore

$$
(T f)(x)=\lim \left(T f_{n_{k}}\right)(x) \text { a.e. }
$$

for a subsequence $\left\{n_{k}\right\}$. This establishes equation (*) for $f$.

The class $C$ contains $C(Y)$ and is a monotone class, therefore it contains $L^{2}(Y, n)$. Thus equation (*) is satisfied for every $f$ in $L^{2}(Y, n)$.

The measures $\mu(x, \cdot)$ can be modified on a subset of $X$ of $m$-measure zero (if necessary) so that $\mu(x, B)$ becomes a Borel function of $x$ for every $B \in \mathscr{B}$ (see Remark 2.2 (vii)).

SECOND PROOF. Let $h_{0}$ be a nonnegative function in the equivalence class $T 1$ and let $\lambda(d x)=h_{0}(x) m(d x)$. First observe that if $h_{0}$ vanishes on the set $S$, then $T f$ vanishes (a.e.) on $S$ for every $f \in L^{2}$ (first use the positivity of $T$ to prove this for $0 \leqslant f \leqslant 1$, then use the linearity and continuity of $T$ ). So we may replace $X$ by $X \backslash S$ and hence we may assume that $h_{0}(x)>0$ for every $x$.

Define a map $\Phi$ of $L^{1}(\lambda)$ into $M(Y)$, the space of bounded Borel measures on $Y$ by the equation

$$
\langle g, \Phi f\rangle=\int f(x)(T g)(x) m(d x) \quad \text { for } g \in C(Y), f \in L^{1}(\lambda) .
$$

Observe that $f T g$ belongs to $L^{1}(m)$ since $|T(\operatorname{Re} g)| \leqslant\|g\|_{\infty} h_{0}$ (a.e.) and $|T(\operatorname{Im} g)|<\|g\|_{\infty} h_{0}$ (a.e.), so

$$
\int|f(x) \operatorname{Tg}(x)| m(d x) \leqslant 2\|g\|_{\infty} \int|f(x)| \lambda(d x) .
$$

Thus $\Phi(f) \in M(Y)$, and $\|\Phi(f)\| \leqslant 2 \int|f(x)| \lambda(d x)$, so $\Phi$ is a bounded operator (it can be shown that $\Phi$ is an isometry, but this fact is not needed).

By the Dunford-Pettis theorem [7, p. 503], there exists a bounded weak*measurable function $x \rightarrow \nu_{x}$ from $X$ into $M(Y)$ such that $\left\|\nu_{x}\right\| \leqslant\|\Phi\|$ for 
every $x$, and

$$
\Phi(f)(\cdot)=\int f(x) \nu_{x}(\cdot) \lambda(d x),
$$

where the integral exists in the weak* sense. So

$$
\langle g, \Phi(f)\rangle=\int f(x) \int g(y) \nu_{x}(d y) \lambda(d x),
$$

and hence

$$
\int(T g)(x) f(x) m(d x)=\iint f(x) g(y) \nu_{x}(d y) h_{0}(x) m(d x),
$$

for all $g \in C(Y)$ and $f \in L^{1}(\lambda)$. Therefore

$$
(T g)(x)=\int g(y) h_{0}(x) \nu_{x}(d y)
$$

for $\lambda$-almost every $x$, and hence for $m$-almost every $x$ since $h_{0}(x)>0$ for every $x$. Now let $\mu(x, d y)=h_{0}(x) \nu_{x}(d y)$, so (*) holds for $f \in C(Y)$. The extension to $L^{2}$-functions follows as before.

REMARK. The preceding theorem is valid (with the same proof) for bounded operators from $L^{p}(Y)$ into $L^{r}(X)$ for $1 \leqslant p<\infty, 1<r \leqslant \infty$.

A kernel $\mu(x, \cdot)$ is called absolutely bounded if $|\mu|(x, \cdot)$ is the kernel of a bounded operator. It is obvious that $\mu$ is absolutely bounded if and only if $\mu=\mu_{1}-\mu_{2}+i\left(\mu_{3}-\mu_{4}\right)$ where each $\mu_{j}$ is positive and is the kernel of a bounded operator. Recall that an operator of $L^{P}(Y, n)$ into $L^{r}(X, m)$ is called order-bounded if for every positive function $u \in L^{P}(Y)$, there exists a positive function $v \in L^{r}(X)$ such that $|(T f)(x)| \leqslant v(x)$ for $m$-almost every $x$ whenever $|f(y)|<u(y)$ for $n$-almost every $y$.

Corollary 3.2. Let $1 \leqslant p<\infty, 1 \leqslant r \leqslant \infty$ and let $T$ be a bounded operator from $L^{p}(Y, n)$ into $L^{r}(X, m)$. The following conditions are equivalent.

(i) $T$ is a pseudo-integral operator with absolutely bounded kernel.

(ii) $T=T_{1}-T_{2}+i\left(T_{3}-T_{4}\right)$ for some order-preserving bounded operators $T_{1}, T_{2}, T_{3}, T_{4}$.

(iii) $T$ is order-bounded.

Proof. The equivalence of (i) and (ii) follows from Theorem 3.1 and the preceding remark, and the equivalence of (ii) and (iii) follows from some general facts about Banach lattices (see, e.g., [19, pp. 229, 233]).

4. Operators with absolutely bounded kernels. Most of the results in this section were proved by Arveson [2] in a slightly less general setting. (The class of operators considered in [2] corresponds to kernels with the property that the total variations $|\mu|(x, Y)$ are uniformly bounded. These kernels are automatically absolutely bounded.) Most of his proofs are still valid in our 
more general setting after slight modifications, and so we will omit details whenever a reference to [2] suffices.

Let $\mu(x, d y)$ be an absolutely bounded kernel (that is, $|\mu|(x, d y)$ is the kernel of some bounded operator). Let $T$ be the operator whose kernel is $\mu(x, d y)$. First we show that $T$ is induced by a measure $\mu$ on the product space $X \times Y$ by the equation

$$
\langle T f, g\rangle=\int f(y) \bar{g}(x) \mu(d x, d y),
$$

where $\langle\cdot, \cdot\rangle$ denotes the inner product in $L^{2}(X, m)$. The measure $\mu$ is defined by the equation

$$
\mu(E)=\iint \chi_{E}(x, y) \mu(x, d y) m(d x)
$$

for every Borel set $E$ in $X \times Y$. To show that this defines a bounded measure, observe that the function $x \rightarrow|\mu|(x, Y)$ belongs to $L^{2}(m)$ and hence to $L^{1}(m)$. An argument similar to the proof of Theorem 2.6 .2 of [4] shows that $\mu$ is a bounded measure (that argument is stated for the case $x \rightarrow|\mu|(x, Y)$ is a bounded function, but extends to our case with no difficulty). A slight modification of the proof of Theorem 2.6.4 of [4] establishes equation (**) for every $f \in L^{2}(n)$ and $g \in L^{2}(m)$.

Now we consider operators given by the equation (**) for some Borel measure $\mu$ on $X \times Y$. First, observe that a necessary condition for (**) to be meaningful is that the integral on the right side must vanish whenever $f=0$ a.e. or $g=0$ a.e. This condition is easily seen to be equivalent to the condition that $\mu$ vanishes on marginally null sets, i.e., Borel sets which are included in rectangles of the form $A \times Y$ or $X \times B$ with $m(A)=0$ or $n(B)=0$. The condition above can be rephrased in a different way. First define the marginal measures $|\mu|_{1}$ and $|\mu|_{2}$ of $|\mu|$ by $|\mu|_{1}(E)=|\mu|(E \times Y)$ for every Borel set $E \subseteq X$ and $|\mu|_{2}(F)=|\mu|(X \times F)$ for every Borel set $F \subseteq Y$. The condition that $\mu$ vanishes on marginally null sets can now be seen to be equivalent to the condition that $|\mu|_{1} \ll m$ and $|\mu|_{2} \ll n$.

If $\mu$ is absolutely continuous with respect to $m \times n$, then $\mu$ satisfies the condition given in the preceding paragraph, but it should be noted that there are measures satisfying that condition but are not absolutely continuous. For example, let $X=Y, m=n$ (assumed to be not purely atomic), and define $\sigma$ on the Borel sets of $X \times X$ by $\sigma(E)=m\{x:(x, x) \in E\}$, then $\sigma$ is concentrated on the diagonal, but $\sigma_{1}=\sigma_{2}=m$.

LEMMA 4.1. Let $\mu$ be a Borel measure on $X \times X$ which vanishes on marginally null sets and has the property that the function $h(x, y)=f(y) \bar{g}(x)$ belongs to $L^{1}(|\mu|)$ whenever $f \in L^{2}(n)$ and $g \in L^{2}(m)$. Then the equation

$$
\left\langle T_{\mu} f, g\right\rangle=\int f(y) \bar{g}(x) \mu(d x, d y)
$$

defines a bounded linear operator $T_{\mu}$ of $L^{2}(Y, n)$ into $L^{2}(X, m)$. 
Proof. Let $f \in L^{2}(Y, n)$. The map $\phi: L^{2}(X, m) \rightarrow C$ defined by

$$
\phi(g)=\int f(y) \bar{g}(x) \mu(d x, d y)
$$

is a conjugate-linear map. To prove that it is bounded, let $g_{n} \rightarrow 0$ and choose a subsequence $g_{n_{i}}$ which converges almost everywhere and which is dominated by a positive function $u$ in $L^{2}(X, m)$ (see 2.2(iii)). By the dominated convergence theorem $\phi\left(g_{n_{i}}\right) \rightarrow 0$. Furthermore, this can be applied to any subsequence of $g_{n}$, to extract a subsequence whose image under $\phi$ converges to zero. Thus $\phi\left(g_{n}\right) \rightarrow 0$ and $\phi$ is bounded. So $\phi(g)=\langle h, g\rangle$ for some $h \in L^{2}(X, n)$. Let $T_{\mu} f=h$, therefore $T_{\mu}$ is a linear map. It remains only to show that it is bounded. Toward this end, let $f_{n} \rightarrow 0$, and take a subsequence $f_{n_{i}}$ which is dominated by a function $u \in L^{2}(Y, n)$ and such that $f_{n_{i}} \rightarrow 0$ almost everywhere. By the dominated convergence theorem

$$
\int f_{n_{i}}(y) \bar{g}(x) \mu(d x, d y) \rightarrow 0 \text { for every } g \in L^{2}(X, m) .
$$

Therefore $T_{\mu} f_{n_{i}} \rightarrow 0$ weakly. By the closed graph theorem $T_{\mu}$ is bounded.

REMARKs. (i) It is obvious that the conditions of Lemma 4.1 are also necessary for $\mu$ to induce a bounded everywhere-defined operator by the equation (**).

(ii) If $\mu$ satisfies the conditions of Lemma 4.1, then so does $|\mu|$.

EXAMPLeS. (1) If $X=Y, m=n$ and $\sigma(E)=m\{x:(x, x) \in E\}$, then $T_{\sigma}=I$. Here $\sigma$ is concentrated on the diagonal $\{(x, x): x \in X\}$.

(2) If $X=Y, m=n$ and $\mu(d x, d y)=\phi(x) \sigma(d x, d y)$ where $\sigma$ is the measure in the preceding example, and $\phi \in L^{\infty}(X)$, then $T_{\mu}=M_{\phi}$, the operator of multiplication by $\phi$. Again $\mu$ is concentrated on the diagonal.

(3) Let $C_{\psi}$ be a bounded composition operator of $L^{2}(Y, n)$ into $L^{2}(X, m)$, so $C_{\psi} f=f \circ \psi$ where $\psi$ is a Borel map of $X$ into $Y$. Then $C_{\psi}=T_{\mu}$ where $\mu(E)=m\{x:(x, \psi(x)) \in E\}$. Here $\mu$ is concentrated on the graph of $\psi$.

(4) By specialization of example 3 we see that all translation operators on $L^{2}(0,1)$ are pseudo-integral operators. These include operators of the form $\left(T_{\alpha} f(x)=f(x+\alpha)\right.$ where $\dot{+}$ is addition modulo 1 and operators of the form $\left(T_{\alpha}^{\prime} f(x)=f(x+\alpha)\right.$ if $0 \leqslant x+\alpha \leqslant 1$ and $\left(T_{\alpha}^{\prime} f\right)(x)=0$ otherwise.

(5) Products $M_{\phi} C_{\psi}$ or $C_{\psi} M_{\phi}$ of a multiplication operator and a composition operator are pseudo-integral operators. The kernel $\mu$ of $M_{\phi} C_{\psi}$ is concentrated on the graph of $\psi$ and is given by $\mu(E)=\int \phi(x) m(d x)$ where the integral is taken over the set $\{x:(x, \psi(x)) \in E\}$. The kernel $\nu$ of $C_{\psi} M_{\phi}$ is also concentrated on the graph of $\psi$ but is given by $\nu(E)=\int \phi(\psi(x)) m(d x)$ where the integral is taken over the set $\{x:(x, \psi(x)) \in E\}$.

Next we show that the class of operator $T_{\mu}$ defined as in Lemma 4.1 is precisely the class of pseudo-integral operators with absolutely bounded kernels. We have already shown that the latter class is included in the former. For the converse we need a result about disintegration of measures. 
LEMMA [2,p. 461]. Let $X$ and $Y$ be standard Borel spaces, and let $\mu$ be a finite positive measure on $X \times Y$, and let $\mu_{1}(A)=\mu(A \times Y)$ be the first marginal measure of $\mu$. Then there exists a map $x \rightarrow \mu_{0}^{x}$ from $X$ into the space of all probability measures on $Y$ such that

(i) The map $x \rightarrow \mu_{0}^{x}(B)$ is a Borel function, for every Borel set $B$ in $Y$.

(ii) $\mu(S)=\int_{X} \int_{Y} \chi_{S}(x, y) \mu_{0}^{x}(d y) \mu_{1}(d x)$ for every Borel set $S \subseteq X \times Y$.

For a proof, see either [1, Theorem 2] or [6, pp. 59-63] together with the remarks on p. 461 of [2].

For measures $\mu$ under consideration (those inducing bounded operators) we give a related disintegration with $m$ in place of $\mu_{1}$.

Proposition 4.2 (SEe [2, Proposition 1.5.3]). Let $\mu$ be a bounded complex Borel measure on $X \times Y$ such that $\mu$ vanishes on marginally null sets (equivalently $|\mu|_{1} \ll m,|\mu|_{2} \ll n$, where $|\mu|_{1}$ and $|\mu|_{2}$ are the marginal measures of $|\mu|)$. Then there exists a map $x \rightarrow \mu^{x}$ of $X$ into the set of all bounded Borel measures on $Y$, and a map $y \rightarrow \mu$ of $Y$ into the set of all bounded Borel measures on $X$, such that

(i) For every Borel sets $A \subseteq X$ and $B \subseteq Y$, the maps $x \rightarrow \mu^{x}(B)$ and $y \rightarrow \mu_{y}(B)$ are Borel functions.

(ii) $\mu(d x, d y)=\mu^{x}(d y) m(d x)$, that is $\mu(S)=\int_{X} \int_{Y} \chi_{S}(x, y) \mu^{x}(d y) m(d x)$, for every Borel set $S$ in $X \times Y$.

(ii) $\mu(d x, d y)=\mu_{y}(d x) n(d y)$.

(iii) $|\mu|(d x, d y)=\left|\mu^{x}\right|(d y) m(d x)=\left|\mu_{y}\right|(d x) n(d y)$.

Moreover $\mu^{x}$ and $\mu$, are essentially unique, i.e. if $\nu^{x}$ and $\nu_{y}$ satisfy (i), (ii), (ii)', and (iii), then $\nu^{x}=\mu^{x}$ a.e. and $\nu_{y}=\mu$, a.e.

For a proof we refer the reader to the proof of Proposition 1.5.3 of [2] which is done under the assumption that $d \mu_{1} / d m$ and $d \mu_{2} / d n$ are bounded, but the argument there is valid in the general case. We give a very brief indication of the proof. Let $|\mu|=\sigma$ and apply the Lemma to $\sigma$ to obtain a disintegration $\sigma(d x, d y)=\sigma_{0}^{x}(d y) \sigma_{1}(d x)$. Note that $\sigma_{1}(d x)=w(x) m(d x)$. Absorb $w$ in $\sigma_{0}^{x}$ to get $\sigma(d x, d y)=\sigma^{x}(d y) m(d x)$. Write $\mu(d x, d y)=$ $\phi(x, y) \sigma(d x, d y)$ and let $\mu^{x}(d y)=\phi(x, y) \sigma^{x}(d y)$.

Next observe that equations (ii) and (ii)' imply that

$$
\begin{aligned}
\int h(x, y) \mu(d x, d y) & =\int_{X} \int_{Y} h(x, y) \mu^{x}(d y) m(d x) \\
& =\int_{Y} \int_{X} h(x, y) \mu_{y}(d x) n(d y),
\end{aligned}
$$

for every $h \in L^{1}(|\mu|)$. To see this, observe that the function $\phi(x)=|\mu|^{x}(Y)$ belongs to $L^{1}(m)$ and apply a slight modification of the argument in $[4, \mathrm{p}$. 101]. 
If $\mu$ induces a bounded operator $T_{\mu}$, then

$$
\left\langle T_{\mu} f, g\right\rangle=\iint f(y) \bar{g}(x) \mu^{x}(d y) m(d x),
$$

and hence

$$
\left(T_{\mu} f\right)(x)=\int f(y) \mu^{x}(d y)
$$

for almost every $x \in X$, that is $T_{\mu}$ is a pseudo-integral operator with kernel $\mu^{x}(d y)$. The same argument applies to $|\mu|$, and since $|\mu|^{x}=\left|\mu^{x}\right|$, we have

$$
\left(T_{|\mu|} f\right)(x)=\int f(y)\left|\mu^{x}\right|(d y) \quad \text { a.e., }
$$

and so $\mu^{x}(d y)$ is an absolutely bounded kernel.

In view of the discussion above, a measure $\mu(d x, d y)$ will be called an absolutely bounded kernel whenever $\mu$ induces a bounded operator. It will be obvious from the context whether $\mu(d x, d y)$ or $\mu^{x}(d y)$ is referred to by the word "kernel".

We now restrict ourselves to the case $X=Y, m=n$, so the operators considered are operators of $L^{2}(X, m)$ into itself.

THEOREM 4.3. If $T$ is a pseudo-integral operator on $L^{2}(X, m)$ with an absolutely bounded kernel, then so is $T^{*}$.

Proof. If $\mu(d x, d y)$ is the kernel of $T$, so $(T f)(x)=\int f(y) \mu^{x}(d y)$ a.e., then

$$
\left(T^{*} f\right)(y)=\int f(x) \bar{\mu}_{y}(d x) \text { a.e. }
$$

where $\bar{\mu}(d x, d y)=\bar{\mu}_{y}(d x) m(d y)$ is the disintegration of $\bar{\mu}$, and the bar denotes complex conjugation.

Next we consider the product of two operators with absolutely bounded kernels. First we describe the product of two measures $\mu$ and $\nu$ which are assumed to be absolutely bounded kernels. This is the same as Arveson's definition [2, p. 463] for the class of measures he describes. Disintegrate $\mu$ and $\nu$ :

$$
\mu(d x, d y)=\mu_{y}(d x) m(d y) \text { and } \nu(d x, d y)=\nu^{x}(d y) m(d x) .
$$

We claim that the functions $y \rightarrow\left|\mu_{y}\right|(X)$ and $x \rightarrow\left|\nu^{x}\right|(Y)$ belong to $L^{2}(X, m)$. For that, observe that $|\mu|$ is the kernel of a bounded operator $S$, and $\left(S^{*} f\right)(y)=\int f(x)\left|\mu_{y}\right|(d x)$ a.e.. Therefore $\left|\mu_{y}\right|(X)=\left(S^{*} 1\right)(y)$ a.e., and hence belongs to $L^{2}(X, m)$. A similar argument proves the same for $\left|\nu^{x}\right|(Y)$. For each $z \in X, \mu_{z} \times \nu^{z}$ defines a bounded measure on $X \times X$. If $w(z)=$ $\left|\mu_{z}\right|(X)\left|\nu^{z}\right|(Y)$, then $w \in L^{1}(X, m)$, and $\left|\left(\mu_{z} \times \nu^{z}\right)(S)\right| \leqslant w(z)$ for every Borel set $S$ in $X \times X$. In particular the function $z \rightarrow\left(\mu_{z} \times \nu^{2}\right)(S)$ belongs to 
$L^{1}(X, m)$. Define $\mu * \nu$ by

$$
(\mu * \nu)(S)=\int_{X}\left(\mu_{z} \times \nu^{z}\right)(S) m(d z)
$$

for every Borel set $S \subseteq X \times X$. The countable additivity of $\mu * \nu$ follows from the dominated convergence theorem.

TheOREM 4.4 (SEe [2, Proposition 1.5.5]). If $\mu$ and $\nu$ are absolutely bounded kernels, then so is $\mu * \nu$ and $T_{\mu * \nu}=T_{\mu} T_{\nu}$.

ProOF. First, we show that $\mu * \nu$ vanishes on marginally null sets. Let $m(E)=0$, then $\left|\mu_{z}\right|(E)=0$ for almost every $z$ since $\int\left|\mu_{z}\right|(E) m(d z)=|\mu|(E$ $\times X)=0$. It follows that $|\mu * \nu|(E \times X) \leqslant \int\left|\mu_{z}\right|(E)\left|\nu^{2}\right|(X) m(d z)=0$. Similarly $|\mu * \nu|(X \times E)=0$.

Next, let $h$ be a Borel function on $X \times Y$, then

$$
\int|h(x, y)|(|\mu| *|\nu|)(d x, d y)=\iiint|h(x, y)||\mu|_{z}(d x)|\nu|^{z}(d y) m(d z),
$$

in the sense that if one side is finite, the other side is also finite and the two are equal. When $h$ is a characteristic function, this is just the definition of $|\mu| *|\nu|$, and the extension to arbitrary functions is done be a standard argument. Therefore if $f, g \in L^{2}(X, m)$, then

$$
\int|f(y)||g(x)|(|\mu| *|\nu|)(d x, d y)=\left\langle T_{|\mu|}^{*}|f|, T_{|\nu|}|g|\right\rangle,
$$

thus $f(y) \bar{g}(x)$ belongs to $L^{1}(|\mu| *|\nu|)$. But the measure $|\mu| *|\nu|$ dominates $|\mu * \nu|$, so $f(y) \bar{g}(x)$ belongs to $L^{1}(|\mu * \nu|)$. By Lemma $4.1, \mu * \nu$ is an absolutely bounded kernel. Furthermore, by Fubini's theorem, we have

$$
\begin{aligned}
\left\langle T_{\mu * \nu} f, g\right\rangle & =\int f(y) \bar{g}(x)(\mu * \nu)(d x, d y) \\
& =\iiint f(y) \bar{g}(x) \mu_{z}(d x) \nu^{z}(d y) m(d z)=\langle F, G\rangle,
\end{aligned}
$$

where $F(z)=\int f(y) \nu^{z}(d y)$ and $G(z)=\int g(x) \bar{\mu}_{z}(d x)$, that is $F=T_{v} f$ and $G=$ $T_{\mu}^{*} g$. Therefore $\left\langle T_{\mu * \nu} f, g\right\rangle=\left\langle T_{\nu} f, T_{\mu}^{*} g\right\rangle=\left\langle T_{\mu} T_{\nu} f, g\right\rangle$, and $T_{\mu, \nu}=T_{\mu} T_{\nu}$.

THEOREM 4.5. The class $\mathcal{P}$ of pseudo-integral operators with absolutely bounded kernels is a selfadjoint algebra containing the identity. The class 9 of integral operators with absolutely bounded kernels is a selfadjoint two-sided ideal in $\mathcal{P}$.

Proof. The first part of the theorem has already been proved. The fact that $\mathcal{G}$ is selfadjoint is proved by an application of Fubini's theorem. It remains only to show that $\mathcal{G}$ is an ideal. Let $\mu$ and $\nu$. be absolutely bounded kernels and assume that $\nu$ is absolutely continuous with respect to $m \times m$. Write $\nu(d x, d y)=k(x, y) m(d x) m(d y)$, so $\nu^{x}(d y)=k(x, y) m(d y)$. Let $S$ be a Borel 
subset of $X \times X$ such that $(m \times m)(S)=0$, and let $S^{x}=\{y:(x, y) \in S\}$, so $S^{x}$ is a Borel set for every $x$, and $m\left(S^{x}\right)=0$ for almost every $x$. Let $E=\left\{x: m\left(S^{x}\right)>0\right\}$, so $m(E)=0$. From the definition of $\mu * \nu$, we have

$$
\begin{aligned}
|\mu * \nu|(S) & \leqslant \iiint \chi_{S}(x, y)|\nu|^{z}(d y)|\mu|_{x}(d x) m(d z) \\
& =\int_{X} \int_{X} \int_{S^{x}}|k(z, y)| m(d y)|\mu|_{z}(d x) m(d z) .
\end{aligned}
$$

The innermost integral vanishes for $x \notin E$, so

$$
\begin{aligned}
|\mu * \nu|(S) & \leqslant \int_{X} \int_{E} \int_{S^{x}}|k(z, y)| m(d y)|\mu|_{z}(d x) m(d z) \\
& \leqslant \int_{X} \int_{X} \int_{E}|k(z, y)||\mu|_{z}(d x) m(d z) m(d y),
\end{aligned}
$$

by Fubini's theorem, and hence

$$
|\mu * \nu|(S) \leqslant \int_{X} \int_{X}|k(z, y)||\mu|_{z}(E) m(d z) m(d y),
$$

but $|\mu|_{z}(E)=0$ for almost every $z$, since $\int|\mu|_{z}(E) m(d z)=|\mu|(E \times X)=0$. Therefore $\left.|k(z, y)| \mu\right|_{z}(E)$ vanishes almost everywhere $(m \times m)$ on $X \times X$, and $|\mu * \nu|(S)=0$. Therefore $\mu * \nu \ll m \times m$, and hence $T_{\mu} T_{\nu}$ is an integral operator. A similar argument (or by taking adjoints) shows that $T_{\nu} T_{\mu}$ is also an integral operator.

Remark. Neither the algebra $\mathscr{P}$ nor the ideal $\mathscr{g}$ is closed in the norm topology since every finite rank operator belongs to $I$ but there are compact operators that do not belong to $\mathscr{P}$ (see Example 2.6).

Question. If $T$ is a compact pseudo-integral operator, must $T$ be an integral operator?

We close this section by giving examples of bounded kernels which are not absolutely bounded.

EXAMPLES. (1) Consider the Hilbert matrix $\left\{a_{m n}\right\}$, where $a_{m n}=(m-n)^{-1}$ if $m \neq n$ and $a_{n n}=0$. This matrix defines a bounded operator on $l^{2}$, but the matrix $\left\{\left|a_{m n}\right|\right\}$ does not give an operator of $l^{2}$ into itself (see [11, pp. 212-214]). This example can be transplanted into $L^{2}(0,1)$ as follows: Let $I_{n}=\left(2^{-n}, 2^{1-n}\right]$ and define a function $k$ on $(0,1) \times(0,1)$ by

$$
k(x, y)= \begin{cases}2^{(n+m) / 2}(m-n)^{-1} & \text { if } x \in I_{m}, y \in I_{n}, m \neq n, \\ 0 & \text { if } x, y \in I_{n} .\end{cases}
$$

Therefore $k$ defines a bounded integral operator $T$ by the equation $(T f)(x)=$ $\int_{0}^{1} k(x, y) f(y) d y$, but $|k|$ does not.

(2) The Fourier transform $F: L^{2}(0,1) \rightarrow l^{2}$ given by $(F f)(n)=$ $\int_{0}^{1} e^{-2 \pi i n x} f(x) d x$ is an integral operator with kernel $k(n, y)=e^{-2 \pi i n y}$, but $|k(n, y)|=1$ and does not give an operator of $L^{2}$ into $l^{2}$. Also $F^{*}$ is not a 
pseudo-integral operator. Again, we can modify this example to get an operator of $L^{2}(0,1)$ into itself by taking $I_{n}=\left(2^{-n}, 2^{1-n}\right]$ and $k(x, y)=$ $2^{n / 2} e^{-2 \pi i n y}$ if $x \in I_{n}$. Therefore $k$ is a bounded kernel, the corresponding operator $T_{k}$ is given by $\left(T_{k} f(x)=2^{n / 2} \hat{f}(n)\right.$ when $x \in I_{n}$. But $k(x, y)$ is not an absolutely bounded kernel since if $f(x) \equiv 1$ and if $g(x)=\int|k(x, y)| f(y) d y$, then $g(x)=2^{n / 2}$ if $x \in I_{n}$, and $\int_{0}^{1}|g(x)|^{2} d x=\int_{n=1}^{\infty} 2^{n} \cdot 2^{-n}=\infty$. We note that $T_{k}^{*}$ is not an integral (or even pseudo-integral) operator.

(3) For $f \in L^{2}(0,1)$, let

$$
\begin{aligned}
k(x, y) & =(1-x)^{-1} \exp (-2 \pi i x y /(1-x)), \\
(T f)(x) & =\int_{0}^{1}(1-x)^{-1} \exp (-2 \pi i x y /(1-x)) f(y) d y,
\end{aligned}
$$

thus

$$
(T f)(x)=(1-x)^{-1} \hat{f}_{0}\left(\frac{x}{1-x}\right),
$$

where $f_{0}$ is the function defined on the real line by $f_{0}(x)=f(x)$ if $0<x<1$ and $f_{0}(x)=0$ otherwise, and $\hat{f}_{0}$ is its Fourier transform. Thus

$$
\begin{aligned}
\int_{0}^{1}|(T f)(x)|^{2} d x & =\int_{0}^{1}(1-x)^{-2}\left|\hat{f}_{0}\left(\frac{x}{1-x}\right)\right|^{2} d x=\int_{0}^{\infty}\left|\hat{f}_{0}(t)\right|^{2} d t \\
& <\int_{-\infty}^{\infty}\left|\hat{f}_{0}(t)\right|^{2} d t=\int_{-\infty}^{\infty}\left|f_{0}(s)^{2}\right| d s=\|f\|^{2} .
\end{aligned}
$$

On the other hand the function $g(x)=\int_{0}^{1}(1-x)^{-1} f(y) d y$ does not belong to $L^{2}(0,1)$ unless $\int_{0}^{1} f(y) d y=0$, so the given kernel is not absolutely bounded. The operator $T^{*}$ is not an integral (or even pseudo-integral operator), since if $\left(T^{*} f\right)(x)=\int_{0}^{1} f(y) \mu(x, d y)$, then by taking functions $f$ which vanish on $(a, 1)$ for some $a<1$, we get

$$
\langle f, T g\rangle=\int_{0}^{a} \int_{0}^{1}(1-x)^{-1} f(x) \bar{g}(y) \exp (2 \pi i x y /(1-x)) d y d x,
$$

and by Fubini's theorem,

$$
\left(T^{*} f\right)(x)=\int_{0}^{a}(1-y)^{-1} f(y) \exp (2 \pi i x y /(1-y)) d y .
$$

Thus $\mu(x, d y)=(1-y)^{-1} \exp (2 \pi i x y /(1-y)) d y$ on $(0, a)$, and since $a$ is arbitrary, $\mu(x, d y)$ is absolutely continuous, and $\mu(x, d y)=\bar{k}(y, x) d y$. But $\bar{k}(y, x)$ is not a bounded kernel, for example if $f(y)=1$, then $\bar{k}(y, x) f(y)$ is not integrable on $(0,1)$.

REMARK. The results of this section are valid (with essentially the same proofs) for operators on $L^{P}(X), 1<p<\infty$. In this case, the adjoint in Theorem 4.3 should refer to the Banach space adjoint acting on $L^{q}(X)$ where $p^{-1}+q^{-1}=1$, and the selfadjointness conclusion of Theorem 4.5 becomes meaningless and should be omitted. 
5. Operators which are always pseudo-integral. Which operators $T$ on Hilbert space $\mathcal{H}$ have the property that every operator unitarily equivalent to $T$ is a pseudo-integral operator? More precisely, given a standard measure space $(X, m)$ that is not purely atomic, we ask when $U T U^{*}$ is a pseudo-integral operator for every unitary operator $U$ from $\mathcal{H}$ onto $L^{2}(X, m)$. Every Hilbert-Schmidt operator is an integral operator (see [18, p. 35]), and so every operator of the form: scalar + Hilbert-Schmidt is always pseudo-integral. We show that these are the only operators with this property. This is a generalization of a theorem of Korotkov [15] which asserts that the unitary orbit of $T$ consists of integral operators if and only if $T$ is Hilbert-Schmidt (see [21] for a proof which lends itself easily to the present situation). The idea is to reduce the problem to the case of a diagonal operator and then use techniques similar to Korotkov's which become very simple in the case of a diagonal operator. Recall that the compression of an operator $T$ to a subspace $\mathscr{N}$ is the operator $P T P \mid \mathscr{T}$, where $P$ is the orthogonal projection onto $\mathscr{R}$. The essential numerical range [7] of an operator $T$ is defined by $\cap W(T+K)^{-}$, where the intersection is taken over the set of all compact operators $K$, and $W(T)^{-}$denotes the closure of the numerical range of $T$. We need the following facts from [7]:

(a) If $\pi$ is the canonical quotient map of $\mathscr{B}(\mathcal{K})$ onto the Calkin algebra, then $W_{e}(T)=V(\pi(T))$, where $V(t)$ denotes the Banach-algebra numerical range of $t$ (see [5, §10]). In particular if $\pi\left(T_{1}\right)$ is unitarily equivalent to $\pi\left(T_{2}\right)$, then $W_{e}\left(T_{1}\right)=W_{e}\left(T_{2}\right)$.

(b) $W_{e}(T)=\{\lambda\}$ if and only if $T-\lambda I$ is compact.

(c) $\lambda \in W_{e}(T)$ if and only if there exists an orthonormal sequence $\left\{e_{n}\right\}$ such that $\left(T e_{n}, e_{n}\right) \rightarrow \lambda$.

The next four lemmas are due to Anderson and Stampfli [24] and to J. P. Williams [25].

LEMMA 5.1. If $P$ is a projection with finite dimensional null space, then $W_{e}(T)=W_{e}(P T P \mid P \mathcal{H})$.

Proof. The operator PTP is a compact perturbation of $T$, so $W_{e}(T)=$ $W_{e}(P T P)$. Let $A=P T P \mid P \mathcal{H}$, and $S$ the unilateral shift, and $n$ the dimension of the null space of $P$, then $S^{n} A\left(S^{*}\right)^{n}$ is unitarily equivalent to $P T P$. But $S$ is essentially unitary, so $W_{e}(A)=W_{e}(P T P)=W_{e}(T)$.

LEMMA 5.2. Every operator has an infinite-dimensional diagonal compression.

Proof. Let $T \in \mathscr{B}(\mathcal{H})$. We will construct an orthonormal sequence $\left\{e_{n}\right\}$ in $\mathcal{H}$ so that the compression of $T$ to the closed linear span of $\left\{e_{n}\right\}$ is diagonal. Choose $e_{1}$ arbitrarily and proceed by induction. Take $e_{n+1}$ to be a unit vector orthogonal to the finite-dimensional space 


$$
\Re_{n}=\bigvee\left\{e_{i}, T e_{i}, T^{*} e_{i}: 1 \leqslant i \leqslant n\right\} .
$$

It is easy to see that $T e_{n}$ is orthogonal to $e_{m}$, whenever $n \neq m$, and hence the compression of $T$ to $\bigvee\left\{e_{n}: n=1,2, \ldots\right\}$ is diagonal.

LEMMA 5.3. Every operator $T$ has a diagonal compression with the same essential numerical range as $T$.

Proof. Let $\left\{\lambda_{n}\right\}$ be a sequence of complex numbers contained in $W_{e}(T)$ such that the limit points of $\left\{\lambda_{n}\right\}$ is $W_{e}(T)$. Modify the proof of the preceding lemma as follows. Choose $e_{n+1}$ so that $\left|\left(T e_{n+1}, e_{n+1}\right)-\lambda_{n+1}\right|<1 /(n+1)$. This is possible since the compression of $T$ to $\Re_{n}^{\perp}$ has the same essential numerical range as that of $T$, therefore we may choose $e_{n+1}$ in $\Re_{n}^{\perp}$ so that $\left|\left(Q T Q e_{n+1}, e_{n+1}\right)-\lambda_{n+1}\right|<1 /(n+1)$ where $Q$ is the projection on $\mathfrak{N}_{n}^{\perp}$. But $\left(Q T Q e_{n+1}, e_{n+1}\right)=\left(T Q e_{n+1}, Q e_{n+1}\right)=\left(T e_{n+1}, e_{n+1}\right)$.

Let $D$ be the compression of $T$ to $\bigvee\left\{e_{n}: n=1,2, \ldots\right\}$, then $D=$ $\operatorname{diag}\left(\alpha_{1}, \alpha_{2}, \ldots\right)$ where $\alpha_{n}=\left(T e_{n}, e_{n}\right)$. By the construction of $e_{n}$, we see that the limit points of $\left\{\alpha_{n}\right\}$ are the same as the limit points of $\left\{\lambda_{n}\right\}$. Therefore $W_{e}(T) \subseteq W_{e}(D)$. The reverse inclusion is always true, so $W_{e}(T)=W_{e}(D)$.

COROLlary 5.4. If $T$ is not of the form: scalar + compact, then $T$ has a compression of the form

$$
\left(\begin{array}{cc}
\alpha I+K_{1} & 0 \\
0 & \beta I+K_{2}
\end{array}\right)
$$

where $\alpha$ and $\beta$ are complex numbers, $\alpha \neq \beta$, and $K_{1}$ and $K_{2}$ are HilbertSchmidt operators.

Proof. Take $\alpha, \beta \in W_{e}(T)$ such that $\alpha \neq \beta$. If $D=\operatorname{diag}\left(\alpha_{1}, \alpha_{2}, \ldots\right)$ is the operator constructed in Lemma 5.3, choose subsequences $\left\{n_{k}\right\}$ and $\left\{m_{k}\right\}$ of the positive integers such that $\left|\lambda_{n_{k}}-\alpha\right|<1 / k$ and $\left|\lambda_{m_{k}}-\beta\right|<1 / k$. Let $\Re_{1}$ and $\Re_{2}$ be the closed linear spans of $\left\{e_{n_{k}}\right\}$ and $\left\{e_{m_{k}}\right\}$ respectively, then the compression of $T$ to $\mathfrak{T}_{1} \oplus \mathfrak{T}_{2}$ has the required form.

Recall that the $s$-numbers of a compact operator $T$ is the nonincreasing sequence $\left\{s_{n}\right\}$ of nonzero eigenvalues of $\left(T^{*} T\right)^{1 / 2}$, counting multiplicities. Recall also that every nontrivial two-sided ideal in $\mathscr{B}(\mathcal{H})$ is contained in the ideal $\mathcal{K}(\mathcal{F})$ of all compact operators. Corresponding to every ideal $G$ there is a set $\delta(\xi)$ of sequences $\left\{\lambda_{i}\right\}$ of nonnegative numbers which are nonincreasing and converge to 0 , such that $T \in \mathcal{G}$ iff the sequence of $s$-numbers of $T$ belongs to $\delta(\mathcal{S})$. Furthermore $\mathcal{S}(\mathcal{G})$ satisfies the following properties:

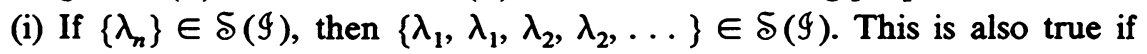
each $\lambda_{i}$ is repeated $m$ times (a fixed $m$ for all $\lambda_{n}$ ).

(ii) $\delta(\xi)$ is closed under addition and multiplication by nonnegative numbers. 
(iii) If $\left\{\alpha_{n}\right\} \in \mathcal{S}(\mathcal{G})$ and if $\left\{\beta_{n}\right\}$ is a nonincreasing sequence of nonnegative numbers with $\beta_{n} \leqslant \alpha_{n}$, then $\left\{\beta_{n}\right\} \in \delta(\mathcal{G})$. For an exposition of these results we refer the reader to p. 26 of [20].

LEMma 5.5. Let $T \in \mathscr{B}(\mathcal{H})$, then $T$ has a diagonal compression $D$ with the property that $D$ belongs to an ideal $G$ if and only if $T \in \mathcal{G}$. In particular $D$ is not a Hilbert-Schmidt operator if $T$ is not.

Proof. If $T$ is not compact, then the result follows from Corollary 5.4. If $T$ is compact, let $\left\{s_{n}\right\}$ be the sequence of $s$-numbers of $T, s_{1}>s_{2} \geqslant \ldots$ We now modify the construction of $\left\{e_{n}\right\}$ in the proof of Lemma 5.2. Choose $e_{1}$ such that $\left|\left(T e_{1}, e_{1}\right)\right| \geqslant \frac{1}{3}\|T\|=\frac{1}{3} s_{1}$. This is possible since the numerical radius $w(T)>\frac{1}{2}\|T\|$. After $e_{1}, \ldots, e_{n}$ have been constructed, let $P_{n}$ to be the projection on $\Re_{n}^{+}$where $\Re_{n}=\bigvee\left\{e_{i}, T e_{i}, T^{*} e_{i}, 1<i<n\right\}$. The operator $T-P_{n} T P_{n}$ has rank at most $6 n$. Therefore $\left\|P_{n} T P_{n}\right\|>s_{6 n+1}$ (see pp. 48-49 of [9]). Choose $e_{n+1}$ so that $\left|\left(T e_{n+1}, e_{n+1}\right)\right|>\frac{1}{3} s_{6 n+1}$. Let $D$ be the compression of $T$ to the closed linear span of $\left\{e_{n}\right\}$, then $D=\operatorname{diag}\left(\alpha_{1}, \alpha_{2}, \ldots\right)$, where $\alpha_{n}=\left(T e_{n}, e_{n}\right)$.

If $T$ belongs to an ideal $\mathcal{G}$, then obviously $D \in \mathcal{G}$. Conversely if $D \in \mathcal{G}$, then $D^{(6)}=D \oplus \cdots \oplus D$ (6 copies) also belongs to $G$, and hence also $3 D^{(6)}$. But the diagonal elements of the latter dominate the sequence $\left\{s_{n}\right\}$, so $T \in 9$.

COROLLARY 5.6. If $T$ is not of the form: scalar + Hilbert-Schmidt, then $T$ has a diagonal compression which is not a scalar + Hilbert-Schmidt.

Proof. Immediate from Lemmas 5.3 and 5.5.

LEMMA 5.7. Let $(X, m)$ be a separable measure space, $Y$ a measurable subset of $X$ such that $L^{2}(Y)$ and $L^{2}(X \backslash Y)$ are both infinite dimensional, and let $\mathcal{H}$ be a separable Hilbert space, $\mathfrak{N}$ a subspace of $\mathcal{K}$ with $\operatorname{dim} \mathfrak{N}=\operatorname{dim} \mathfrak{K}^{\perp}=\infty$, $A$ an operator on $\mathcal{X}$ and $B$ the compression of $A$ to $\Re$. If $U A U^{*}$ is a pseudo-integral operator on $L^{2}(X)$ for every unitary operator $U$ from $\mathcal{H}$ onto $L^{2}(X)$, then $V A V^{*}$ is a pseudo-integral operator on $L^{2}(Y)$ for every unitary operator $V$ from $\mathcal{X}$ onto $L^{2}(Y)$.

Proof. Let $V$ be a unitary operator of $\mathscr{N}$ onto $L^{2}(Y)$ and let $U$ be any unitary operator of $\Re^{\perp}$ onto $L^{2}(X \backslash Y)$, and $W=V \oplus U$. By hypothesis $W A W^{*}$ is a pseudo-integral operator, so

$$
\left(W A W^{*} f\right)(x)=\int_{X} f(t) \mu(x, d t) \text { a.e. }
$$

If $\nu(x, \cdot)$ is the restriction of $\mu(x, \cdot)$ to $Y$, then

for almost every $y$ in $Y$.

$$
\left(V A V^{*} f\right)(y)=\int_{Y} f(t) \nu(y, d t)
$$


THEOREM 5.8. Let $(X, m)$ be a standard measure space which is not purely atomic, and let $T$ be an operator on Hilbert space $\mathcal{H}$. Then UTU $U^{*}$ is a pseudo-integral operator on $L^{2}(X, m)$ for every unitary operator $U$ from $\mathcal{H}$ onto $L^{2}(X, m)$ if and only if $A$ is of the form: scalar + Hilbert-Schmidt.

Proof. The "if" part is trivial. To prove the "only if" part assume that $U A U^{*}$ is always pseudo-integral for every unitary $U$. By Corollary 5.6 and Lemma 5.7 we need only consider diagonal operators and measure spaces $(Y, m)$ with no atoms. Without loss of generality we may take $m(Y)=1$. Since every standard Borel space is Borel isomorphic to a complete separable metric space [17], we may assume that $Y$ is a complete separable metric space, and by a result in [18, p. 327], there is a Borel subset $Y_{0}$ of $Y$ with $m\left(Y_{0}\right)=0$, a Borel subset $E_{0}$ of $[0,1]$ of Lebesgue measure zero and a Borel equivalence $\psi$ of $Y \backslash Y_{0}$ onto $[0,1] \backslash E_{0}$ such that $m\left(\psi^{-1}(S)\right)=\lambda(S)$ where $\lambda$ is Lebesgue measure on $[0,1]$. Therefore we may take $Y=[0,1]$ with Lebesgue measure. (Note that we may add or delete a set of $m$-measure zero, since if $\mu(x, d y)$ is a kernel of an operator and $E$ a set of $m$-measure zero, then $\{x:|\mu|(x, E)>0\}$ is a set of $m$-measure zero, cf. Remark 2.2(ii).)

First we show that $T$ must be a scalar + compact. By Corollary 5.4 we need only show that an operator of the form: $\left(\begin{array}{ll}\alpha I & 0 \\ 0 & \beta I\end{array}\right)+$ Hilbert-Schmidt $(\alpha \neq \beta)$ is not always pseudo-integral. Since every scalar and every HilbertSchmidt operator is always pseudo-integral, we need only consider $\left(\begin{array}{ll}I \\ 0 & 0\end{array}\right)$. But this is unitarily equivalent to the projection of $L^{2}$ onto $H^{2}$ which is not pseudo-integral (see Example 2.4).

So $T=\lambda I+K$ where $K$ is compact. We will show now that $K$ is HilbertSchmidt. The operator $K$ also has the property that $U K U^{*}$ is pseudo-integral for every unitary $U: \mathcal{K} \rightarrow L^{2}(X)$. By adding a Hilbert-Schmidt operator we may assume that $K=\left(\begin{array}{ll}D & g \\ 0 & 0\end{array}\right)$ where $D=\operatorname{diag}\left(\lambda_{1}, \lambda_{2}, \ldots\right)$. Now choose a lacunary sequence $\left\{n_{k}\right\}$ of positive integers, that is $\left(n_{k+1} / n_{k}\right) \geqslant a>1$, and let $\phi_{n}(t)=\exp (-2 \pi i n t)$, and define an operator $K_{0}$ on $L^{2}(0,1)$ by

$$
\begin{aligned}
& K_{0} \phi_{n_{k}}=\lambda_{k} \phi_{n_{k}} \text { for } k=1,2, \ldots, \\
& K_{0} f=0 \text { if } f \perp \phi_{n_{k}} \text { for all } k .
\end{aligned}
$$

This extends to a bounded operator on $L^{2}(0,1)$ which is unitarily equivalent to $K$. Therefore $K_{0}$ is a pseudo-integral operator, i.e.,

$$
\left(K_{0} f\right)(t)=\int f(s) \mu(t, d s) \text { a.e. }
$$

Let $E_{0}$ be a set of measure zero such that

$$
\begin{aligned}
& |\mu|(t, \cdot)<\infty \text { for } t \notin E_{0}, \\
& \left(K_{0} \phi_{n}\right)(t)=\int \phi_{n}(s) \mu(t, d s) \text { for } t \notin E_{0}, \text { and } n \in Z .
\end{aligned}
$$


Fix $t \in[0,1] \backslash E_{0}$ and let $\nu(d s)=\mu(t, d s)$, thus $\nu$ is a bounded Borel measure, and

$$
\begin{aligned}
\hat{\nu}(n) & =\int \phi_{n}(s) \nu(d s)=\left(K_{0} \phi_{n}\right)(s) \\
& = \begin{cases}\lambda_{k} \phi_{n_{k}}(t) & \text { if } n=n_{k}, \\
0 & \text { otherwise. }\end{cases}
\end{aligned}
$$

Since $\hat{\mu}(n)=0$ for all negative $n$, the F. and M. Riesz theorem [12, p. 47] implies that $\mu$ is absolutely continuous, and hence $\Sigma \lambda_{k} \exp \left(-2 \pi i n_{k} t\right) \exp (2$ $\left.\pi n_{k} x\right)$ is the Fourier series of a function $g(x)$ in $L^{1}(0,1)$. By [13, p. 109], we must have $\Sigma\left|\lambda_{k}\right|^{2}<\infty$, i.e. $K$ is Hilbert-Schmidt.

REMARKS. 1. A different proof of Theorem 5.8 can be obtained by slightly modifying the argument used in [21] for integral operators. That proof is more elementary (no results from Fourier series are used), and is valid on any separable sigma-finite measure space (not necessarily standard).

2. Another interesting question is this: which operators $T$ have the property that some operator unitarily equivalent to $T$ is pseudo-integral? The answer is: all operators. This follows from a result of Weidmann [23, Satz 7.3] which implies that if 0 belongs to the right essential spectrum of $T$, then $T$ is unitarily equivalent to an integral operator.

6. Operators whose restriction to $L^{\infty}$ is pseudo-integral. Theorem 2.7 gives a necessary condition for an operator from $L^{2}(Y, n)$ into $L^{2}(X, m)$ to be a pseudo-integral operator. We ask if this condition is also sufficient. The answer is no. It turns out, however, that the condition of Theorem 2.7 characterizes a related class of operators, namely the operators $T$ where

$$
(T f)(x)=\int f(y) \mu(x, d y) \text { a.e. }
$$

for every $f$ in $L^{\infty}(Y, n)$. It should be noted that it is possible for the operator $T$ to be bounded and for (*) to be valid for $f$ in a dense subset but not for every $f$ in $L^{2}$ (see Examples 6.2). A simple example (on a sigma-finite measure space) is the Fourier transform $f \rightarrow \hat{f}$, from $L^{2}(-\infty, \infty)$ onto itself, which is defined by the equation

$$
\hat{f}(x)=(2 \pi)^{-1 / 2} \int_{-\infty}^{\infty} f(y) e^{-i x y} d y
$$

for $f \in L^{1} \cap L^{2}$, and then extended to a unitary operator on all of $L^{2}(-\infty, \infty)$. If $f \in L^{2}$ but $f \notin L^{1}$, the integral on the right side is not defined. 
THEOREM 6.1. Let $T$ be a bounded operator from $L^{2}(Y, n)$ into $L^{2}(X, m)$. The following conditions are equivalent:

(i) The restriction of $T$ to $L^{\infty}(Y)$ is a pseudo-integral operator.

(ii) There exists a positive Borel function $\Omega$ on $X$ such that

$$
|T f(x)| \leqslant\|f\|_{\infty} \Omega(x) \text { a.e. }
$$

for $f \in L^{\infty}$.

Proof. If $T \mid L^{\infty}$ is a pseudo-integral operator with kernel $\mu(x, d y)$, then $|\mu|(x, Y)$ must be finite for almost every $x$, since the constant function 1 belongs to $L^{\infty}(Y, n)$ and hence must be $|\mu|(x, \cdot)$-integrable for almost every $x$. So condition (ii) is satisfied if we take $\Omega(x)=|\mu|(x, Y)$. (If $|\mu|(x, Y)=\infty$, take $\Omega(x)=0$.)

To prove the converse, we may assume that $Y$ is a compact metric space (see [17]). Assume that $T$ satisfies (ii) and let $E_{n}=\{x: n-1 \leqslant \Omega(x)<n\}$. For every $g \in L^{1}\left(E_{n}, m\right)$, define a linear functional $\phi_{g}$ on $C(Y)$ by

$$
\phi_{g}(f)=\int(T f)(x) g(x) m(d x) .
$$

This is a bounded functional since

$$
\left|\phi_{g}(f)\right| \leqslant\|f\|_{\infty} \int_{E_{n}} \Omega(x)|g(x)| m(d x) \leqslant n\|f\|_{\infty}\|g\|_{1} .
$$

Therefore, there is a bounded Borel measure $\lambda_{g}$ on $Y$ such that $\left\|\lambda_{g}\right\| \leqslant n\|g\|_{1}$, and

$$
\left\langle f, \lambda_{g}\right\rangle=\int(T f)(x) g(x) m(d x)
$$

for every $f \in C(Y)$.

The map $g \rightarrow \lambda_{g}$ is a bounded linear operator of $L^{1}\left(E_{n}\right)$ into $M(Y)$, the space of bounded Borel measures on $Y$. By the Dunford-Pettis Theorem,

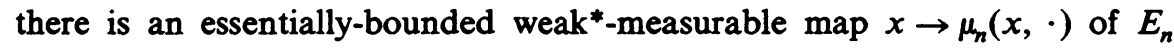
into $M(Y)$ such that

$$
\text { ess } \sup \left|\mu_{n}\right|(x, Y) \leqslant n, \quad \lambda_{g}(\cdot)=\int g(x) \mu_{n}(x, \cdot) m(d x),
$$

where the integral exists in the weak*-topology, i.e.,

$$
\left\langle f, \lambda_{g}\right\rangle=\int g(x) \int f(y) \mu_{n}(x, d y) m(d x),
$$

for every $f \in C(Y)$ and every $g \in L^{1}\left(E_{n}, m\right)$. From (1) and (2), we have

$$
\int_{E_{n}}(T f)(x) g(x) m(d x)=\int_{E_{n}} g(x) \int_{Y} f(y) \mu_{n}(x, d y) m(d x) .
$$


Since this is valid for every $g \in L^{1}\left(E_{n}, m\right)$, we must have

$$
(T f)(x)=\int_{Y} f(y) \mu_{n}(x, d y)
$$

for almost every $x$ in $E_{n}$. Now let

$$
\mu(x, \cdot)=\mu_{n}(x, \cdot) \text { if } x \in E_{n},
$$

thus $x \rightarrow \mu(x, \cdot)$ is a weak ${ }^{*}$-measurable function from $X$ into $M(Y)$, and for $f \in C(Y)$,

$$
(T f)(x)=\int f(y) \mu(x, d y) \text { a.e. }
$$

For $f \in L^{\infty}$, there is a sequence of continuous functions $\left\{f_{n}\right\}$ converging to $f$ almost everywhere and dominatedly, that is, $\left|f_{n}\right| \leqslant g \in L^{\infty}$. The validity of (*) for $f \in L^{\infty}$ follows from the dominated convergence theorem.

The Borel measurability of $\mu(x, B)$ for every Borel subset $B$ of $Y$ follows from the weak*-measurability of the map $x \rightarrow \mu(x, \cdot)$ as in Remark 2.2 (vi).

REMARK. As can be seen from the proof, the theorem is valid under the assumption that $T$ is merely a linear transformation from $L^{\infty}(Y)$ into the linear space of (equivalence classes of) measurable functions on $X$ such that for every sequence $\left\{f_{n}\right\}$ converging to $f$ almost everywhere and dominatedly, the sequence $\left\{T f_{n}\right\}$ converges to $T f$ is some sense (e.g., in measure, or almost everywhere, or in the weak*-topology). See Examples below.

EXAMPLES 6.2. We give two pseudo-integral operators from $L^{\infty}$ into $L^{2}$. One of the operators is unbounded in the $L^{2}$-norm, the other is bounded, but the integration formula defining $T f$ on $L^{\infty}$ is not valid for all $f \in L^{2}$.

(a) Let $X=Y=(0,1)$ with Lebesgue measure and let $k(x, y)=y^{-1 / 2}$. For every $f \in L^{\infty}$, and every $x$, the function $k(x, \cdot) f(\cdot)$ is integrable and the equation

$$
(T f)(x)=\int_{0}^{1} k(x, y) f(y) d y
$$

defines a linear transformation of $L^{\infty}$ into $L^{2}$. Here $|(T f)(x)|<2\|f\|_{\infty}$. It is easy to see that $T$ is not bounded in the $L^{2}$-norm.

(b) Let $X=Y=(0,1)$ with Lebesgue measure and

$$
k(x, y)=x^{-1 / 2} y^{-1 / 2} \exp (-i \log x \log y) .
$$

If $f \in L^{\infty}$, then $\int_{0}^{1}|k(x, y) f(y)| d y \leqslant 2\|f\|_{\infty} x^{-1 / 2}$, so the equation

$$
g(x)=\int_{0}^{1} k(x, y) f(y) d y
$$

defines a measurable function $g(x)$. We will show that $g \in L^{2}$ and that $\|g\|_{2}<M\|f\|_{2}$ for some constant $M$, so the map $f \rightarrow g$ extends to a bounded linear operator from $L^{2}$ into itself. By a change of variable we see that 


$$
\begin{aligned}
g\left(e^{-s}\right) & =\int_{0}^{\infty} e^{s / 2} e^{-t / 2} f\left(e^{-t}\right) e^{-i s t} d t \\
& =\sqrt{2 \pi} e^{s / 2} \hat{F}(s), \quad s>0,
\end{aligned}
$$

where

$$
F(t)= \begin{cases}e^{-t / 2} f\left(e^{-t}\right) & \text { for } t>0 \\ 0 & \text { for } t<0\end{cases}
$$

and $\hat{F}$ is the Fourier transform of $F$. But

$$
\int_{0}^{1}|f(t)|^{2} d t=\int_{0}^{\infty} e^{-x}\left|f\left(e^{-x}\right)\right|^{2} d x=\int_{-\infty}^{\infty}|F(s)|^{2} d s
$$

and

$$
\begin{aligned}
\int_{0}^{1}|g(x)|^{2} d x & =\int_{0}^{\infty} e^{-s}\left|g\left(e^{-s}\right)\right|^{2} d s=2 \pi \int_{0}^{\infty}|\hat{F}(t)|^{2} d t \\
& \leqslant 2 \pi \int_{\infty}^{\infty}|\hat{F}(t)|^{2} d t=2 \pi \int_{-\infty}^{\infty}|F(s)|^{2} d s
\end{aligned}
$$

by Plancherel's formula. Thus $\|g\|_{2} \leqslant \sqrt{2 \pi}\|f\|_{2}$. Finally we show that there is a function $f$ in $L^{2}$, for which the function $k(x, \cdot) f(\cdot)$ is not integrable for any $x$ in $(0,1)$. Take $f(y)=y^{-1 / 2} /(1-\log y)$.

REMARK. Theorem 6.1 is similar to the characterization of Carleman integral operators given by Korotkov [14] and Weidmann [23, Satz 2.9]. They show that $T$ is a Carleman integral operator (i.e., the kernel $k(x, y)$ is an $L^{2}$-function of $y$ for almost every $x$ ) if and only if there exists a positive Borel function $\Omega$ on $X$ such that $|(T f)(x)| \leqslant\|f\|_{2} \Omega(x)$, a.e. for every $f$ in $L^{2}$. Furthermore $T$ is a Hilbert-Schmidt operator if and only if $\Omega$ can be chosen in $L^{2}(X)$ (see [23, Korollar 2.10]).

7. Sigma-finite measure spaces. Until now, we have dealt only with finite measure spaces $(X, m)$ and $(Y, n)$. In this section we make some remarks about extensions to sigma-finite measure spaces. When $m$ and $n$ have total finite variations, the kernel $\mu(x, \cdot)$ must also have finite total variation for almost every $x$ since the function 1 belongs to $L^{2}(Y, n)$ and hence must be integrable with respect to almost all of the measures $|\mu|(x, \cdot)$. In the case $m$ or $n$ have infinite total variation we need "measures" $\mu(x, \cdot)$ which are allowed to have finite total variations and are complex-valued. Since there is no such a thing, we define the kernel $\mu(x, \cdot)$ to be the "product" $w(x, y)|\mu|(x, d y)$ where $w(x, y)$ is a complex-valued function with modulus 1 which is a Borel function of $y$ for almost every $x$, and where $|\mu|(x, \cdot)$ is a sigma-finite positive measure on the Borel subsets of $Y$. The integral $\int f(y) \mu(x, d y)$ will be understood to mean $\int f(y) w(x, y)|\mu|(x, d y)$. The measurability condition of the map $x \rightarrow \mu(x, B)$ in Definition 2.1 would have to be 
modified into the following: For every Borel subset $B$ of $Y$ of finite $n$ measure, the map $x \rightarrow \mu(x, B)$ is a Borel function. We note that for such a set $B$, the total variation $|\mu|(x, B)$ must be finite for almost every $x$ since $\chi_{B} \in L^{2}(Y)$. With this as our definition of a "kernel" and with the corresponding definition of a pseudo-integral operator, most of the results of the preceding sections remain valid in the new setting with slight modifications of the proofs. We make a few remarks about the exceptions.

For operators with absolutely bounded kernels, we have

$$
\langle T f, g\rangle=\int f(y) \bar{g}(x) \mu(d x, d y),
$$

where now $\mu(d x, d y)$ is the product $\phi(x, y)|\mu|(d x, d y)$ of a unimodular jointly-measurable function $\phi$ and a positive sigma-finite measure $|\mu|$. It is easy to see that $\mu$ must be sigma-finite in a very special way: there are disjoint Borel sets $X_{n}$ (respectively $Y_{n}$ ) in $X$ (respectively $Y$ ) such that $X=\cup X_{n}$, $Y=\cup Y_{n}$, and $|\mu|\left(X_{n} \times Y_{m}\right)<\infty$ for every $n$ and $m$. We call a measure with this property bi-sigma-finite. All of the results of $\$ 4$ now extend to the new setting.

Theorems 2.7 and 6.1 must be modified. The appropriate extension of Theorem 6.1 should be as follows:

TheOREM. Let $Y=\cup Y_{n}$, where $\cup Y_{n}$ is an increasing sequence of Borel subsets of $Y$ of finite measure and let $T$ be a bounded operator from $L^{2}(Y)$ into $L^{2}(X)$. The following conditions are equivalent.

(i) The restriction of $T$ to $L^{\infty}\left(Y_{n}\right)$ is a pseudo-integral operator for every $n$.

(ii) There is a sequence $\Omega_{n}$ of positive Borel functions on $X$ such that for every $f$ which vanishes outside $Y_{n}$, we have

$$
|(T f)(x)| \leqslant\|f\|_{\infty} \Omega_{n}(x) .
$$

The proof is omitted.

\section{REFERENCES}

1. M. B. Abrahamse and T. L. Kreite, The spectral multiplicity of a multiplication operator, Indiana Univ. Math. J. 22 (1973), 845-857.

2. W. Arveson, Operator algebras and invariant subspaces, Ann. of Math. (2) 100 (1974), 433-532.

3. ___ An invitation to $C^{*}$-algebras, Springer-Verlag, New York, 1976.

4. R. B. Ash, Real analysis and probability, Academic Press, New York, 1972.

5. F. F. Bonsall and J. Duncan, Complete normed algebras, Springer-Verlag, New York, 1973.

6. N. Bourbaki, Eléménts de mathématique, Livre VI: Intégration. Chapitre 6: Intégration vectorielle, Hermann, Paris, 1959.

7. N. Dunford and J. T. Schwartz, Linear operators, Part I, Interscience, New York, 1958.

8. P. A. Fillmore, J. G. Stampfli and J. P. Williams, On the essential mumerical range, the essential spectrum, and a problem of Halmos, Acta Sci. Math. (Szeged) 33 (1972), 179-192.

9. I. C. Gohberg and M. G. Krein, Theory and applications of Volterra operators in Hilbert space, Transl. Math. Monographs, vol. 24, Amer. Math. Soc., Providence, R.I., 1969. 
10. P. R. Halmos, A Hilbert space problem book, Van Nostrand, New York, 1967.

11. G. H. Hardy, J. E. Littlewood, and G. Polya, Inequalities, Cambridge Univ. Press, London, 1934.

12. K. Hoffman, Banach spaces of analytic functions, Prentice-Hall, Englewood Cliffs, N.J., 1962.

13. Y. Katznelson, An introduction to harmonic analysis, Wiley, New York, 1968.

14. V. B. Korotkov, Integral operators with Carleman kernels, Dokl. Akad. Nauk SSSR 165 (1965), 1496-1499.

15. __ Strong integral operators, Siberian Math. J. 16 (1974), 1137-1140.

16. K. Kuratowski, Topology, Vol. I, Academic Press, New York, 1966.

17. G. Mackey, Borel structure in groups and their duals, Trans. Amer. Math. Soc. 85 (1957), 265-311.

18. H. L. Royden, Real analysis, 2nd ed., Macmillan, New York, 1968.

19. H. H. Schaefer, Banach lattices and positive operators, Springer-Verlag, New York, 1974.

20. R. Schatten, Norm ideals of completely continuous operators, Springer-Verlag, Berlin, 1960.

21. A. R. Sourour, $A$ note on integral operators (submitted).

22. V. S. Sunder, Unitary orbits of integral operators, Notices Amer. Math. Soc. 24 (1977), A-639.

23. J. Weidmann, Carlemanoperatoren, Manuscripta Math. 2 (1970), 1-38.

24. J. Anderson and J. G. Stampfli, Commutators and compression, Israel J. Math. 10 (1971), 433-441.

25. J. P. Williams, The essential numerical range, unpublished manuscript, 1971.

Department of Mathematics, Untverstiy of Toronto, Toronto, Ontario, Canada M5S 1A1

Current address: Department of Mathematics, University of Guelph, Guelph, Ontario, Canada NIG 2W1 